\title{
Wenn viele Wege aus dem Fenster führen - Konzeptuelle Variation im Bereich von Bewegungsereignissen
}

\author{
Raphael Berthele (Freiburg i. Ue.)
}

\begin{abstract}
In this contribution, different varieties of German (Swiss German dialects, Standard High German) are compared with regard to their conceptual and syntactic elaboration of spatial descriptions. The case study examines a particular scene from the informant's picture-book based narratives, a scene wich refers to a dog climbing onto a window sill and then falling out of the window. The analysis shows considerable variability with respect to the linguistic means used to describe the scene, and the author argues that there are corresponding differences in the cognitive construal of the spatial event. The data show consistently that the Swiss German dialects tend to be more detailed in their elaboration of the path of the moving figure (e.g., the dog can be construed as falling "into" the window "out"), whereas speakers of Standard High German tend to use a generic, semantically and syntactically simpler construction. The analysis of this particular scene is demonstrated to coincide with a generally observable tendency of Swiss German dialects: Within motion verb predicates, the constituents encoding spatial arguments tend to be both syntactically and semantically enriched.
\end{abstract}

\section{$1 \quad$ Einleitung}

In der Variationslinguistik liegt das Augenmerk traditionellerweise auf der Vielfalt der Formen, mit denen verschiedene Gewährspersonen (Gwpp.) oder Varietäten dieselben Inhalte ausdrücken können. Variation der phonetisch-phonologischen, der morphologischen und seit neuestem auch der syntaktischen Form sind und werden für das Schweizerdeutsche umfassend erforscht und in z.T. vorbildlicher Weise in Atlanten, Grammatiken und Wörterbüchern aufbereitet. Ein in der Variationslinguistik weniger prominenter Aspekt sind Unterschiede im Bereich dessen, was in der kognitiven Linguistik oft construal (Langacker 1999: 206) genannt wird - Unterschiede in der mentalen Konstruktion und Erfassung eines propositionalen Zusammenhanges und dessen Überführung in eine sprachliche Äusserung.

Sicherlich finden sich in der dialektologischen Literatur gerade im für den vorliegenden Beitrag wichtigen Bereich der Raum-Ausdrücke immer wieder Hinweise etwa auf z.T. extrem elaborierte Systeme der Raumreferenz (cf. z.B. die zahlreichen Beiträge in Rowley 1980), und damit auch auf eine verschieden geartete, in unterschiedlichem Ausmass elaborierte konzeptuelle Erfassung von räumlichen Konstellationen. Auch erhielt in den letzten Jahren 
die Diskussion um sprachliche Relativität neuen Aufwind (cf. etwa Levinson 1996, Niemeier/Dirven 2000, Werlen 2002). In der Regel interessieren sich Arbeiten im letztgenannten Problemfeld für typologisch sehr unterschiedliche Sprachen, deren fundamental unterschiedliche grammatische Systeme und Lexika sodann Unterschieden im Bereich der kognitiven Erfassung der "Welt" entsprechen. Prominentes Beispiel für die Untersuchung dieses Zusammenhanges von sprachtypologischen Charakteristika und kognitiven Korrelaten sind die Arbeiten im Bereich der Bewegungsverben, oftmals etikettiert mit Slobins Begriff Thinking for Speaking (cf. Slobin 1991, 1996). Diese Arbeiten zeigen, dass SprecherInnen von Sprachen, die unterschiedliche Lexikalisierungsmuster zeigen, nicht nur unterschiedliche rhetorische Stile haben, sondern beim Versprachlichen der Inhalte auch unterschiedliche Vorstellungen zugrundelegen. Der vorliegende Beitrag profitiert sowohl methodisch als auch konzeptuell von diesen Arbeiten, allerdings mit einem in zweierlei Hinsicht veränderten Fokus: Einerseits geht es hier um die Untersuchung von typologisch sehr ähnlichen "Sprachen", nämlich verschiedenen Varietäten des Deutschen, und andererseits sollen hier nicht die Bewegungsverben, sondern die Konstituenten in der Schwesterposition des Verbs analysiert werden. Anhand einer Szene einer Bildergeschichte, die von Gewährspersonen aus verschiedenen Dialektregionen nacherzählt wurde, wird ein meines Wissens bisher nicht beschriebenes Beispiel für konzeptuelle Variation innerhalb des Schweizerdeutschen (und zwischen Schweizerdeutsch und der hochdeutschen Standardsprache) beschrieben. Der Beitrag wird zeigen, dass für die Beschreibung exakt desselben visuellen Stimulus syntaktisch und lexikalisch verschiedene sprachliche Muster verwendet werden. Ein Makro-Ereignis (der Fall eines Hundes aus dem Fenster) wird auf der Mikroebene unterschiedlich beschrieben, und zu einem gewissen Grad auch unterschiedlich konzeptualisiert, insbesondere dort, wo das Bewegungsereignis in unterschiedliche Teilaspekte und Segmente zerlegt wird. An diesem Beispiel soll paradigmatisch aufgezeigt werden, dass die untersuchten schweizerdeutschen Varietäten im Vergleich zur Standardsprache zu einem offeneren Ausdruck der räumlichen Semantik neigen.

Die Daten, die der folgenden Analyse zugrunde liegen, wurden von verschiedenen ExploratorInnen gesammelt, grossmehrheitlich im Verlauf der Jahre 2001-2003. ${ }^{1}$ Sie gehören $\mathrm{zu}$ einem Korpus, das in Rahmen eines Projekts $\mathrm{zu}$ raumlinguistischen Aspekten verschiedener Varietäten des Deutschen sowie benachbarter romanischer Sprachen erarbeitet und ausgewertet wurde und wird (Berthele [in Vorbereitung], für Teilresultate siehe Berthele 2004, Berthele [im Druck]). Tabelle 1 gibt eine Übersicht über die in diesem Beitrag berücksichtigten Varietäten. Die Gewährspersonen gehören (mit Ausnahme des Zürichdeutschen) verschiedenen Altersgruppen an, sind jedoch alle in ihren jeweiligen Dialekträumen gut verwurzelt, und gewährleisten in diesem Sinne tatsächlich regionaltypische Ausdrucksweisen.

\footnotetext{
${ }^{1}$ Die zürichdeutschen Froschgeschichten wurden früher zu einem anderen Zweck gesammelt, ich danke Regula Schmidlin, dass sie mir diese zur Verfügung gestellt hat. Die restlichen Froschgeschichten wurden mehrheitlich von mir erhoben, einige wurden von einigen meiner StudentInnen im Rahmen eines Kurses zum Thema Raumlinguistik an der Universität Freiburg/Fribourg erhoben. Mein Dank geht deshalb auch an Michael Ehrler, Martin Klopfenstein, Marlene Schild, Susanne Schneider und Denise Pfammatter.
} 


\begin{tabular}{|l|r|}
\hline \multicolumn{1}{|c|}{ Idiom } & n Gwpp. \\
\hline Standardsprache (STD) & 20 \\
\hline Berndeutsch (BED) & 10 \\
\hline Muotathal (MUD) & 26 \\
\hline Senslerdeutsch (SED) & 10 \\
\hline Walliserdeutsch (WSD) & 4 \\
\hline Zürichdeutsch (ZHD) & 8 \\
\hline
\end{tabular}

Tabelle 1: Anzahl Gewährspersonen pro Varietät

Die Gewährspersonen wurden gebeten, eine - aus der psycholinguistischen Forschung bekannte - wortlose Bildergeschichte (Mayer 1969) nachzuerzählen. Die Geschichte handelt von einem Jungen und seinem Hund auf der Suche nach einem entwischten Frosch. Wie schon kurz angesprochen liegt das besondere Augenmerk des vorliegenden Beitrags auf einer Szene am Anfang dieser Geschichte. Auf der Suche nach dem entwischten Frosch sieht man auf einem Bild die beiden Protagonisten der Geschichte, einen Jungen und seinen Hund, am Fenster des Zimmers des Jungen. Auf dem nächsten Bild sieht man, wie der Hund vom Fensterbrett stürzt, um, auf dem dritten hier relevanten Bild, auf dem Boden draussen zu landen. Beim Sturz aus dem Fenster steckt der Kopf des Hundes in einem Glas, das ehemals dem Frosch als Heim gedient hatte, und dieses Glas zerschlägt beim Aufprall auf dem Boden. Dieser Ausschnitt ist eine von verschiedenen Szenen in der Geschichte, wo plötzliche und schnelle Bewegung (besonders in abwärts-Richtung) dargestellt ist. Sie ist, wie dieser Beitrag zeigen sollte, eine der Passagen, wo sich das Spektrum von möglichen Ausdrucksweisen der verschiedenen DialektsprecherInnen sehr gut zeigen lässt. Nach einer kurzen Einführung in die Theorie (2) werden die verschiedenen sprachlichen Varianten vorgeführt und diskutiert (3). Abschliessend (4) wird gezeigt, dass diese herausgegriffene Episode für generelle Tendenzen im Sinne einer Mikrotypologie von Standardsprache und Dialekten steht.

\section{Theorie und Terminologie}

Um die Variation im Bereich von Bewegungsereignissen beschreiben zu können, sind einige raumlinguistische Grundbegriffe unabdingbar. Diese sollen hier darum kurz eingeführt werden. Ich stütze mich dabei terminologisch überwiegend auf Arbeiten aus der Psycholinguistik (Becker 1994) und aus der kognitiven Semantik (Langacker 1999; Talmy 2000a und 2000b).

Bewegungsereignisse werden als zwei Objekttypen sowie der zwischen letzteren bestehenden Relation konzeptualisiert (vgl. Bsp. 1).

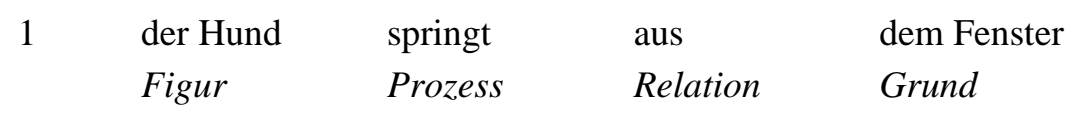

Das Objekt, auf welches der Diskurs primär fokussiert ist und dessen Bewegung durch den Raum (oder auch deren statische Position im Raum) beschrieben wird, wird Figur (figure)

\footnotetext{
${ }^{2}$ Die Gwpp. kommen aus folgenden Städten/Regionen: Berlin, Hamburg, Lübeck, München, Siegen, Tübingen
} 
genannt. ${ }^{3}$ Bei Bewegungsereignissen bewegt sich die Figur relativ zu ihrer Umgebung, und die für die Konzeptualisierung relevanten Teile dieser Umgebung werden Grund (ground) genannt:

The Figure is a moving or conceptually movable entity whose site, path, or orientation is conceived as a variable the particular value of which is the relevant issue. The Ground is a reference entity, one that has a stationary setting relative to a reference frame, with respect to which the Figure's site, path, or orientation is characterized. (Talmy 2000a: 184)

Die konzeptuelle Struktur eines Ereignisses beinhaltet nach Talmy ausserdem einen Aktivierungsprozess. Dieser ist nach Talmy per definitionem im Verb lexikalisiert.

Die Relation, die zwischen Figur und Grund besteht, nennt Talmy die Assoziationsfunktion (association function). Bei sich bewegenden Figuren entspricht diese Funktion dem Weg (path), den die Figur im Verlauf ihrer Bewegung zurücklegt, bei statischen Figuren ist entspricht diese Assoziationsfunktion dem Verhältnis von Eigenort der Figur zum sie umgebenden Raum.

In der kognitiven Linguistik wird oft davon ausgegangen, dass Konzepte des Typs Relation in bildhafter Weise gespeichert sind, jeweils in einem sog. image schema (Johnson 1987). Solche Schemata sind einfache grundlegende Konzepte, die das Wahrnehmen der uns umgebenden physischen (und metaphorisch dann auch sozialen) Welt strukturieren. Ein zentrales Schema im hier relevanten Zusammenhang ist dasjenige von source-path-goal (Quelle-Weg-Ziel; cf. Langacker 1999: 55) - eine Bewegung hat schematisch gesehen einen Ausgangspunkt und führt über einen Weg zu einem Ziel. Das Quelle-Weg-Ziel-Schema beinhaltet a priori zwei Grundelemente, Quelle und Ziel, weitere Grundelemente können jedoch integriert werden, etwa sogenannte Meilensteine, d.h. saliente Grundelemente, die als Zwischenstationen den Weg der Figur situieren helfen.

Eigenorte der Figur, aber auch Quell- und Zielobjekte sind oft Schemata topologischer Natur, d.h. sie beinhalten Relationen wie Innensein oder Aussensein, jedoch keine Informationen bezüglich gewisser räumlicher Eigenschaften wie Grössenverhältnisse und Raumachsen:

In der allgemeinen Topologie geht es um die Abbildung von Punktmengen unter Wahrung bestimmter Zusammenhangsverhältnisse. Man denke sich Punktmengen aus elastischem Material bestehend. Bei elastischer Verformung bleiben bestimmte Eigenschaften erhalten, z.B. innere Punkte bleiben innere Punkte, benachbarte Punkte bleiben benachbart. Bei dieser Betrachtungsweise werden geometrische Eigenschaften oder Grösseneigenschaften von Figuren ausgeblendet. Dem entspricht die Vorstellung von Teilräumen. Ihre Zuordnung zu Objekten ist nicht an starre Formen oder Entfernungen gebunden, sondern ist orientiert an der Gleichartigkeit von Elementen. (Becker 1994: 13f.)

Typische image schemas, die topologischer Natur sind, sind also Konzepte wie Innenraum (und damit auch Aussenraum) oder Rand. Die Assoziationsfunktion verortet in der Regel die

\footnotetext{
${ }^{3}$ Wo Talmy von figure und ground spricht, finden sich bei Langacker nahezu synonym die Begriffe trajector und landmark (cf. Langacker 1990: 10). In der deutschen Psycholinguistik findet sich manchmal das Begriffspaar Thema und Relatum (z.B. in Becker 1994). Da sich im Bereich des frog story research Talmys Terminologie durchgesetzt hat, soll diese auch hier verwendet werden.
} 
Figur relativ zu solchen topologischen Teilräumen, sowohl bei statischen (der Frosch ist im Glas) als auch bei dynamischen Ausdrücken (der Frosch springt aus dem Glas). Dabei werden jedoch topologische Teilräume oft mit achsenbezogenen und damit nichttopologischen Konzepten verbunden (z.B. die vertikale Achse in der Frosch ist auf/unter dem Tisch).

Das Finitum kann ausser dem schematischen Prozess zusätzliche Informationen, etwa solche die Art oder andere Begleiterscheinungen der Bewegung betreffend. Talmy (2000b: 222) spricht hier von Co-Ereignissen. Das Ausmass, in welchem solche Co-Ereignisse in die Verben verpackt werden, sowie die Eigenschaft, auch Weg-Informationen im Verb auszudrücken, hat einen grossen Teil der sprachvergleichenden Diskussion um Bewegungsverben dominiert (vgl. hierzu etwa Talmy 1985; Slobin 1991, 1996, Özcaliskan/Slobin 1999, Berthele 2004). Diese Diskussion spielt in unserem Zusammenhang hier jedoch nur eine untergeordnete Rolle. Entscheidend ist, dass die räumliche Relation, also das, worum es im Lokalisierungsausdruck in zentraler Weise geht, im Deutschen in den Präpositionen sowie in der von der Präposition regierten NP steht. Um die konzeptuelle Variation im Bereich der Raumrelationen wird es im Folgenden vordringlich gehen. Die hier nur sehr verkürzend mögliche Darstellung der Begrifflichkeiten sollte als Instrumentarium zur Analyse der dialektalen Variation im Bereich der Raumrelationen ausreichen.

\section{Die verschiedenen Arten, aus dem Fenster zu fallen}

In diesem Abschnitt sollen zunächst die verschiedenen Varianten des Aus-dem-FensterFallens dargestellt werden. Nicht alle dieser Varianten kommen in allen untersuchten Varietäten vor, und sie treten auch in sehr unterschiedlichen Frequenzen auf. Doch sollen die Frequenzen und Verteilungen hier vorerst keine Rolle spielen, vielmehr sollen die verschiedenen Varianten der Versprachlichung dargestellt werden.

Vorweg muss noch bemerkt werden, dass hier nur jene Gwpp. berücksichtigt wurden, die dieses Ereignis überhaupt sprachlich wiedergeben. In einigen wenigen narrativen Texten wird es nämlich übergangen oder nur implizit ausgedrückt. Dies kommt jedoch nur selten vor, denn das Ereignis ist verhältnismässig dramatisch und salient im Vergleich zu anderen auf den Bildern dargestellten Vorgängen.

\subsection{Der einfache Weg, aus dem Fenster zu fallen}

In diesem Abschnitt werden zuerst die Fälle behandelt, denen ein verhältnismässig einfaches mentales Bild zugrunde liegt: die Figur bewegt sich auf einem Weg weg von der Quelle. Die Quelle kann dabei als Container oder einfach als neutraler "Ort" konstruiert sein.

Eine "Standardversion" der Fenstersturz-Episode, wie sie ganz ähnlich von den meisten deutschen Gwpp. produziert wird, ist in 2 wiedergegeben. 
und während er sich vorbeugte, um auch nach Ferdinand zu suchen, fiel er STD:MK5 ${ }^{4}$ kopfüber aus dem Fenster

In 2 wird der Weg in der Präposition aus ausgedrückt, es handelt sich dabei um einen Weg, dessen Quelle ein Container (und damit topologisch ein in-Raum) ist, aus dem sich die Figur hinausbewegt. Das Grundelement in der von P regierten NP, das Fenster, wird somit entweder selber als Container konzeptualisiert, oder das Fenster steht metonymisch für den ganzen Container. Letztere Variante ist naheliegend, handelt es sich doch beim Fenster um jenen Teil des Rands des Containers, an dem die Figur den Container verlässt - und solche salienten Teile einer Domäne sind es, die für Metonymien bevorzugt herangezogen werden (Lakoff 1999: 514).

Eine Alternative, die jedoch von den standardsprachlichen SprecherInnen nur gerade in einem Fall gewählt wird, ist die um ein Richtungsadverb ergänzte Konstruktion wie in 3.

3 und er fällt aus dem Fenster hinaus

STD:RB38

Das Richtungsadverb wiederholt einerseits die bereits in der P lexikalisierte topologische Relation, zusätzlich kommt hier aber noch die für viele Varietäten des Deutschen typische Angabe der "Bewegungsperspektive" hinzu; in diesem Fall also die Bewegung weg vom deiktischen Zentrum, welches sich hier an der Quelle der Bewegung im Innern des Zimmers befindet (zur Geschichte und Dialektologie dieser Richtungsadverbien cf. Hinderling 1980).

Die nächsten in diesem Abschnitt zu behandelnden Varianten konstruieren die Quelle nicht als Container, sondern noch schematischer als eine Art "Ort ohne Eigenschaften". Die Belege dieses Typs sind streng genommen nicht direkt vergleichbar mit den restlichen Formen, da die gesamte Szene von den ErzählerInnen anders - wohl auch präziser - wiedergegeben wird (vgl. Beleg 4). versuchte es ihm gleichzutun, sass auf dem Fensterbrett, und versuchte, auch nach dem Frosch zu rufen, allerdings hatte er ja dieses Glas auf dem Kopf, und als er so damit beschäftigt war, rutschte er aus und fiel vom Fensterbrett im ersten Stock auf den Boden.

Was bei den meisten anderen Gwpp. in einen einfachen Satz integriert wird (und somit nach Auffassung der kognitiven Semantik als ein Ereignis konzeptualisiert wird, cf. Talmy 2000b: 212), wird hier zerlegt in eine (auf den Bildern nicht sichtbare) Bewegung hin zum Fenster, eine statische Lokalisierung des Hundes "auf dem Fensterbrett", die eine vorausgegangene Aufwärtsbewegung der Figur impliziert, sowie schliesslich eine Bewegung vom Fensterbrett

\footnotetext{
${ }^{4}$ Die Belege werden (vor dem Doppelpunkt) mit einem Kürzel für die Varietät sowie (nach dem Doppelpunkt) mit einem Kürzel für die befragte Gewährsperson identifiziert. Für die Entschlüsselung der Varietätenkürzel siehe Tabelle 1.
} 
wieder hinunter. Mit der $\mathrm{P}$ von wird die Bewegung weg von einem dimensional oder topologisch nicht weiter spezifizierten Ort, der Quelle, angegeben.

Im Schweizerdeutschen findet sich eine ähnliche Lösung mit der $\mathrm{P} a b$ (Beleg 5). Die Semantik der $\mathrm{P} a b$ jedoch umfasst zusätzlich zur Bewegung weg von einer (topologisch neutralen) Quelle auch noch den Aspekt der Abwärts-Bewegung.

Ein letzter hier zu nennender Typ ist die Verwendung der P durch wie in Bsp. 6. Allen in diesem Abschnitt behandelten Belegen ist gemeinsam, dass, innerhalb eines Bewegungsereignisses, die Bewegung der Figur mittels eines einfachen Weges charakterisiert wird. Dieser Weg kann entweder nur mittels topologischer Räume definiert sein oder aber zusätzlich einen Achsen-Aspekt miteinschliessen.

\subsection{Zwei Wege: Der etwas kompliziertere Weg, aus dem Fenster hinaus zu fallen}

In diesem Abschnitt werden die Belege besprochen, in denen die Fenstersturz-Szene nicht mit einem einfachen Weg beschrieben wird, sondern mit einem aus mindestens zwei Teilen zusammengesetzten Weg.

Nachdem wir in Beleg 3 und 4 gesehen haben, dass eine Möglichkeit, die Szene zu beschreiben, die Quelle der Bewegung profiliert, sehen wir in Beleg 7, dass diese Möglichkeit auch ausgebaut werden kann. Zur Angabe der Quelle tritt hier ein Richtungsadverb, das zusätzlich die Abwärtsrichtung der Bewegung codiert. ${ }^{5}$

7 ja u nä hee si grüeft $\mathrm{u}$ zum fenschter usiggogget aber niene isch der frosch

BED:MK4 gsii/da uf z mau isch do der hund vom fenschtersims ache kchiit ja und dann haben sie gerufen und zum Fenster hinausgekuckt aber nirgends ist der Frosch gewesen/da auf ein Mal ist da der Hund vom Fenstersims hinunter gefallen

Die Syntax solcher Belege ist unklar (Wunderlich 1984: 79; Olsen 1996: 306; Eisenberg et al. 1998: 399 und ausführlich Steinitz 1969). Grob zusammengefasst ergeben sich die folgenden

\footnotetext{
${ }^{5}$ Ich bin nicht ganz sicher, ob das Richtungsadverb hier tatsächlich eine deiktische Perspektive codiert oder nicht. Die Gwp. ist dem Berner Oberland zuzuordnen, einer Region, die gemäss SDS VI: 111 die Unterscheidung zwischen hinab und herab kennt. Ache (<ab-her) entspräche damit semantisch dem standardsprachlichen herab, was in diesem Kontext eher unwahrscheinlich ist. Wie Hinderling (1980) zeigt, unterliegen diese deiktischen Richtungsadverbien ohnehin ständiger "semantischer Erosion", was auch für den oft inkonsequenten Gebrauch der Formen in den alpinen Schweizer Varietäten verantwortlich sein dürfte.
} 
Analysevarianten: Entweder gehen wir von einem komplexen Verb aus (9), dem eine PP als Adjunkt (Angabe) hinzugefügt wird, oder wir gehen von einem einfachen $\mathrm{V}$ mit einer komplexen PP aus (8).

$8 \quad$...dass der Hund [[vom Fenster runter] fällt $]$

$9 \quad$...dass der Hund [[vom Fenster] [runter-fällt]]

Im letzteren Fall ist die interne Struktur der PP ambig, entweder bildet das ADV runter einen leeren Kopf, der von einer PP modifiziert wird, oder die P von ist der Kopf einer PP, die von einem ADV modifiziert wird. In Bsp. 7 wird die Figur zuerst (implizit) beim Fenster situiert, und dann wird auf den Teil des Fensters gezoomt, wo der Junge und der Hund sich befinden. Anschliessend wird die Bewegung als von der Quelle - dem Fensterbrett - weg beschrieben. Wie schon oben angesprochen, sind die Belege dieses Typs den anderen hier präsentierten Varianten nicht völlig äquivalent, denn die Gwpp., die diese Variante wählen, engen den Referenzbereich ihrer Äusserungen ein und referieren nicht auf das Fenster als Ganzes, sondern nur auf den Teil, auf dem die Protagonisten sitzen und von dem aus der Hund nach draussen springt oder fällt.

Beleg 10 enthält ebenfalls ein Richtungsadverb, das den nach unten gerichteten Weg ausdrückt - dieses Adverb codiert jedoch zusätzlich auch die hin-Richtung.

WSD:DP4 da - ups- ist auf einmal der Bello durch das Fenster hinunter-gefallen

Ausserdem codiert die $\mathrm{P}$ hier den Weg der Figur durch eine Begrenzung hindurch. Das Fenster wird hier eventuall gar nicht - wie im Zusammenhang mit Bsp. 2 oben erwogen metonymisch für einen Teil des Raumes genommen, sondern als isolierte Entität, die die Figur durchquert. Auch in 11 wird das Fenster als ein durchquerter Raum konstruiert, allerdings nun zusätzlich mit einem Adverb, das raus bedeutet.

11 und jetzt schpringt er dänk dr $\mathrm{z}$ fäischter uuse

MUD:RB35 und jetzt springt er halt durch das Fenster raus

Die Figur verändert in diesem Beispiel ihren Eigenort im Bezug auf zwei topologische Aspekte: erstens durchquert sie ein Grund-Element, und zweitens verlässt sie den in-Raum.

Auch die nächste Variante codiert den Ortswechsel von innen nach draussen, diesmal ist diese Assoziationsfunktion jedoch wieder in der Präposition ausgedrückt. In einer parallel dazu konstruierten zweiten PP wird dann nachgedoppelt, dass der Weg auf den Aussenraum hin gerichtet ist. Diese Konstruktion ist zweifellos redundant, denn sie drückt den raus-Weg zweimal aus. Sie ist syntaktisch komplexer als die Konstruktionen in Kap. 0 oben. Ob dieser syntaktischen Komplexität auch eine semantische Komplexität entspricht, ist fraglich, in der Regel wird in der Literatur hier von einer semantischen "Verdeutlichung" der Relation 
ausgegangen (vgl. etwa McIntyre 2001: 9, Stadelmann 1978: 216). Syntaktisch komplexer ist zweifellos auch die wiederum redundante Fügung in 12, mit einer ersten PP mit dem FensterGrundelement und einer zweiten PP mit dem "Grundelement" (im weitesten Sinne) des Aussenraumes in einem Pronominaladverb.

In der nächsten Variante wird der Weg der Figur zweigeteilt, und hier erhöht sich nun zweifellos auch die konzeptuell-semantische Feinauflösung des Beweungsereignisses. In der $\mathrm{P}$ wird eine Bewegung in einen topologischen in-Raum (offensichtlich das Fenster) konzeptualisiert. Erinnern wir uns, dass topologische Räume dimensionsneutral sind (siehe oben, Kapitel 0) - eine Fensterscheibe mit ihrer quasi zweidimensionalen mentalen Repräsentation kann also durchaus als mit einem Innenraum versehen aufgefasst werden. In einem zweiten Schritt wird wiederum der hier bereits oft angetroffene raus-Weg konzeptualisiert.

$13 \mathrm{u}$ de frosch fritzli springt ids feischter usi

SED:SS3

und der Frosch Fritzli springt ins Fenster hinaus

Da in den ausgewerteten Bewegungsverbdaten die Konstruktion von Beispiel 13 nur gerade einmal vorkommt (vgl. unten, Tabelle 2), muss man sich fragen, ob es sich hier um eine Idiosynkrasie handelt. Dies kann jedoch ausgeschlossen werden, wenn wir die zu Bewegungsverben syntaktisch und begrifflich parallelen Perzeptionsverben hinzunehmen. Perzeptionsverben können als eine Art "fiktive Bewegungsverben" aufgefasst werden: Zwar bewegt sich keine Figur entlang eines Weges, doch wird die Wahrnehmungsleistung beschrieben als Weg einer aktiven Entität entlang des Weges, der zwischen dem Wahrnehmer (oft Experiencer genannt) und dem Wahrgenommenen (Experienced) liegt (vgl. Talmy 2000a: 115f.). Bsp. 14 und 15 zeigen also, dass die analoge Konstruktion im Bereich der Perzeptionsverben von zwei der sechs Sensler Gwpp. verwendet wird. ${ }^{6}$

14 si gugge ids feischter usi

SED:SS5 sie kucken ins Fenster hinaus der Nettu kuckt ins Fenster hinaus mit seinem Wasserglas

Um eine Idiosynkrasie handelt es sich dagegen wohl bei der letzten hier darzustellenden Variante. Ich habe sie im gesamten Material nur ein einziges Mal gefunden, und im Gegensatz zu den anderen in diesem Beitrag dargestellten Konstruktionen, die alle

\footnotetext{
${ }^{6}$ Auch bei Verben des Sprechens zeigen sich Parallelen mit den Bewegungskonstruktionen (vgl. etwa na hends usem Fäischter use glärmet, MUD:ME3)
} 
nachvollziehbar sind, findet sich hier keine Interpretation, die dem Referenzbereich, also dem Fall eines Hundes aus einem Fenster, angemessen scheint. fällt der Bello gerade samt dem Glas noch äh auf das Fenster hinab

Der Weg wird hier in einen auf- und einen hinunter-Weg aufgeteilt. Letzteren kennen wir aus verschiedenen anderen Konstruktionen, er bereitet uns keine Bauchschmerzen. Rätselhaft ist der $a u f$-Weg, der hier nicht angemessen scheint. Allenfalls könnte man die Konstruktion "retten", indem man bedenkt, dass es in vielen schweizerdeutschen Mundarten nach wie vor üblich ist, die Reise in eine bestimmte Ortschaft mit auf X (also etwa uf Züri, uf Bärn, etc.) anzugeben. Vielleicht ist es diese Verwendung von auf als richtungsgebende Präposition, die diese Gwp. zur in 16 angegebenen Konstruktion motiviert hat. Doch angesichts der schlechten Beleglage und des auf Wortfindungsprobleme oder Ähnliches hinweisenden Verzögerungssignals [æ:] sollten wir dieser Variante nicht zuviel Gewicht beimessen.

\subsection{Ein Ort und ein Weg: Wenn man beim Fenster rausfällt}

In diesem Abschnitt werden diejenigen Konstruktionen beschrieben, in denen nicht ein zweiteiliger Weg, sondern eine Kombination eines Ortes und eines Weges konzeptualisiert wird.

Die erste hier zu nennende Fügung lokalisiert die Figur in der Nähe des Grundes. Mit Becker (1994) müssen wir bei solchen Näherelationen zwischen der Regio und der Peripherie unterscheiden: Einen neutralen topologischen Ort, einen unspezifischen Raum, in dem sich die Figur befindet, nennen wir Regio. Die Regio entspricht einem ,gestalt- und dimensionsneutralen Konzept“" (Becker 1994: 42), das gleichzeitig Eigenort und Einflussbereich des Grundes ist. Einen Raum dagegen, der an den Eigenort des Grundobjektes angrenzt, nennen wir Peripherie. Bei einer Lokalisierung mittels der P bei nun handelt es sich nicht um einen Ort in der Regio, sondern um einen Ort in der Peripherie, als in einem Raum mit deutlicherem Bezug zum Grundelement.

$17 \mathrm{u}$ dernaa flügt der hund bem fääschter usi ahi uf $\mathrm{z}$ glaas

BED:MK3 und danach fliegt der Hund beim Fenster hinaus hinunter auf das Glas nachher haben sie beim Fenster hinausgekuckt und um Hilfe gerufen

Der eigentlich Weg wird im Adverb geliefert, es ist wiederum ein Weg aus dem In-Raum hinaus, mit einem deiktischen Element, das die Perspektivierung angibt (usi vs. usa; zu diesen perspektivierenden Richtungsadverbien vgl. SDS VI: 105-112). In 17 wird die VP noch zusätzlich ergänzt durch ein weiteres Richtungsadverb (ahi=hinab) sowie durch eine zweite PP. Insgesamt werden hier also drei Weg-Teile angegeben: hinaus (im Bezug auf Quelle), 
hinunter (im Bezug auf Quelle) und auf (im Bezug auf Ziel). ${ }^{7}$ Wiederum finden sich hier Beispiele mit Perzeptionsverben, die streng parallel sind zu den Bewegungsverben (18).

Ein Grenzfall ist die zweite hier zu behandelnde Konstruktion. Hier wird die $\mathrm{P} z u$ in Kombination mit einem Richtungsadverb verwendet (vgl. Beleg 19). Es ist fraglich, ob es sich hierbei tatsächlich um eine $\mathrm{P}$ handelt, die die Richtung angibt. Die Konstruktion zu+NP:Dat+ADV codiert sicherlich als Ganze einen Weg, wobei die semantische Hauptlast der Relationsbedeutung auf dem ADV liegt. Die P scheint mir hier fast nur noch als Markierung für ein Grundelement (Quelle oder allenfalls eine Art "frühen" Meilenstein) zu fungieren. Belege 21 und insbesondere 22 sollte diese Annahme illustrieren helfen. ist jetzt der Hund, der Hund zum Fenster raus gesprungen der Frosch geht - hüpft zum Glas raus

Man wäre vielleicht versucht anzunehmen, dass hier der Weg der Figur in einen $z u$-Abschnitt, also einen Weg hin zum Grundelement, und einen raus-Abschnitt, also einen Weg aus dem Container zergliedert wird. Wenn aber $z u$ einen Weg angeben würde, wäre 20 unsinnig, befindet sich doch der Frosch schon seit längerer Zeit im Glas und geht nicht erst dorthin, um dann hinauszuspringen. Die $\mathrm{P} z u$ gibt hier also nicht einen Weg, sondern einen topologischen Ort im Sinne der Regio an. ${ }^{8}$

Dass beide in diesem Abschnitt behandelten Konstruktionen tatsächlich einen Ort und einen Weg kombinieren, belegt auch der Umstand, dass das Adverb jeweils nicht weggelassen werden kann, da sonst die dynamische Wegsemantik erlischt (vgl. Bspp. 19 und 20).

\section{$21 *$ der Hund fliegt beim Fenster \\ 22 ?der Hund fliegt zum Fenster}

Satz (22) ist nur scheinbar einen Gegenbeleg, da es sich, wie oben diskutiert, bei der $\mathrm{P} z u$ in Beispiel 19 und 20 oben nicht um eine richtungsangebende $\mathrm{P}$ handeln kann, sondern um eine ortsangebende.

\footnotetext{
${ }^{7}$ Dies ist typisch für die gleichzeitig kurze und präzise Erzählweise der Gwp. MK3 (sein Geschichte zählt zu den kürzesten überhaupt, enthält aber weit mehr Details zu Grundelementen und Wegen als längere Geschichten anderer Gwpp.).

${ }^{8}$ Während Becker in ihrer Untersuchung solche neutralen Lokalisierungen für das Englische, Französische und Türkische beschreibt, finden sich in ihren Daten keine Beispiele aus dem Deutschen. Dies dürfte damit zusammenhängen, dass die in den Froschgeschichten gefundene Verwendung von $z u$ in der Standardsprache nicht mehr produktiv ist, sie findet sich vor Allem in den hier beschriebenen Konstruktionen mit einem FensterGrundelement, aber auch in zunehmend veraltenden Formen wie der Dom zu Speyer, zu Wasser und zu Land, etc. (vgl. Duden 1995, Bd. 8: 4031)
} 


\section{Frequenzen}

Die in Kapitel 0 dargestellten Varianten sind nicht alle gleich häufig, und sie kommen auch nicht alle in allen Varietäten vor. Tabelle 2 gibt einen Überblick, welche Konstruktionen wie häufig in der Standardsprache und in den Dialekten auftreten. Um die Häufigkeiten und den Stellenwert der einzelnen Konstruktionstypen abschätzen zu können, müssen sie natürlich in Relation zur Gesamtzahl der Fenstersturz-Episoden in der jeweiligen Varietät gesehen werden (vgl. letzte Tabellenzeile). Gewisse Konstruktionen kommen in unseren Daten nur in jeweils einem Dialekt vor (etwa die in Bsp. 17 oben wiedergebene), doch ist die Datenbasis zu klein, um hier kategorische Aussagen zum Auftreten bestimmter Typen zu machen. Tendenzen lassen sich jedoch klar ablesen. Diese Tendenzen sollen sodann in diesem Abschnitt wiederum verglichen werden mit einer den Resultaten einer Korpusanalyse von geschriebenen standardsprachlichen Texten.

\begin{tabular}{|l|r|r|}
\hline & Standardsprache & Dialekte \\
\hline $\mathrm{ab}$ & 14 & 1 \\
\hline aus & & 5 \\
\hline durch & 1 & 2 \\
\hline von & 3 & 12 \\
\hline aus-raus & & 1 \\
\hline auf-runter & 1 & 1 \\
\hline aus-runter & & 1 \\
\hline bei-raus & & 2 \\
\hline durch-raus & & 1 \\
\hline durch-runter & & 1 \\
\hline in-raus & 2 & 2 \\
\hline von-runter & & 16 \\
\hline zu-raus & $21^{9}$ & $45^{10}$ \\
\hline Total Fenster-Belege & & \\
\hline
\end{tabular}

Tabelle 2: Häufigkeiten der Varianten

Die standardsprachlichen Daten zeigen weniger Konstruktionstypen als die Dialektdaten. Ausserdem tendiert der Dialekt ganz klar zur Verteilung der Raumsemantik auf verschiedene Partikel, während die Standardsprache den einfachen Ausdruck des Wegs bevorzugt. Tabelle 2 zeigt hier deutlich, dass die einfache Variante mit dem einfachen aus-Weg in den standardsprachlichen Froschgeschichten dominiert (14 Belege von 21). Dieselbe Variante kommt auch im Schweizerdeutschen vor, wenn auch nicht im selben Ausmass. Die zweithäufigste Konstruktion ist die des Typs zum Fenster hinaus. Diese kommt jedoch in den hier ausgewerteten Daten nur im Dialekt vor, wo sie auch die häufigste ist, sie kommt aber in der Standardsprache nicht vor. Eine Analyse von Korpora geschriebener deutscher

\footnotetext{
${ }^{9}$ Da eine Gwp. die Szene zweimal schildert, ist hier die Summe höher als die Anzahl Gwpp. für die deutsche Standardsprache.

10 Da nicht alle Gwpp. der Dialekte die Fensterszene explizit schildern, ist die Summe hier kleiner als die Gesamtzahl der Gwpp.
} 
Standardsprache (vgl. Tabelle 3) zeigt jedoch, dass sie auch in standardsprachlichen Texten von deutschen AutorInnen gebraucht wird - allerdings viel seltener als die einfache ausKonstruktion.

\begin{tabular}{|r|l|r|r|}
\hline & Typ & absolut & relativ \\
\hline 1 & zum Fenster /s1 hinaus/raus & 178 & 0.10 \\
\hline 2 & aus dem Fenster /s1 hinaus/raus & 23 & 0.01 \\
\hline 3 & aus dem Fenster \%s1 hinaus/raus & 1665 & 0.89 \\
\hline
\end{tabular}

Tabelle 3: Anzahl Hits für ausgewählte Fenster-Konstruktionen im COSMAS-Korpus; ${ }^{11}$ Zeile 1: "zum Fenster" und im gleichen Satz "hinaus" oder "raus"; Zeile 2: "aus dem Fenster" und im gleichen Satz "hinaus" oder "raus"; Zeile 3: "aus dem Fenster" und nicht "hinaus" oder "raus" im gleichen Satz

In Zeile 1 von Tabelle 3 findet sich die absolute und relative Vorkommenshäufigkeit der zum Fenster hinaus/raus-Konstruktion. Diese Konstruktion ist im Schweizerdeutschen insgesamt am häufigsten, in den COSMAS-Korpora der geschriebenen Sprache ${ }^{12}$ machen sie ungefähr $10 \%$ aus. Zeile 2 zeigt sodann, dass die Konstruktion mit dem den Weg verdoppelnden Adverb extrem selten sind (ungefähr 1\%). In Zeile 3 schliesslich wird die Vorkommenshäufigkeit des Strings "aus dem Fenster" gezählt, jeweils unter Ausschluss des Wortes "hinaus" oder "raus" im selben Satz. Diese einfachen Konstruktionen machen also fast 90\% der Belege in diesem Korpus aus. Dies ist genau die Konstruktion, die auch in den hier ausgewerteten mündlichen Nacherzählungen in der Standardsprache dominiert. Die KorpusSuche nach den übrigen in Abschnitt 0 oben dargestellten Konstruktionen blieb ergebnislos. Es zeigt sich also klar, dass das Standarddeutsche zur einfachen Konstruktion aus dem Fenster neigt - sowohl in den hier ausgewerteten Froschgeschichten als auch im Korpus geschriebener Sprache. Innerhalb der schweizerdeutschen Varietäten tut sich ein breites Spektrum verschiedenster Konstruktionen auf, wobei die von den Gwpp. der Standardsprache präferierte Konstruktion möglich, aber aber statistisch marginal ist. Wenn wir die in den Daten vorgefundenen Konstruktionen grob in zwei Kategorien aufteilen, den durch eine einzige Partikel ausgedrückten einfachen Weg einerseits und die Kombination von Partikeln andererseits, so ergeben sich die in Tabelle 4 angegebenen Verteilungen.

\begin{tabular}{|r|r|r|}
\hline$\%$ & Standard & Nonstandard \\
\hline einfach & $71 \%$ & $18 \%$ \\
\hline komplex & $29 \%$ & $82 \%$ \\
\hline
\end{tabular}

Tabelle 4: einfache und komplexe Wege in Standardsprache und Dialekten

Tabelle 4 gibt die Anteile an der Gesamtmenge der relevanten Konstruktionen an. Es zeigt sich hier noch mal in aller Deutlichkeit, dass die Standardsprache die einfache Konstruktion

\footnotetext{
11 http://corpora.ids-mannheim.de/ cosmas/

12 Die Suche innerhalb der COSMAS-Korpora gesprochener Sprache ergab so wenige Hits (2 für "zum Fenster raus/hinaus/naus", 2 für "aus dem Fenster"), dass daraus keine Schlüsse gezogen werden können. Es liegt auf der Hand, dass die in diesem Beitrag behandelte Fragestellung auch für diatopische und diastratische Variation der Varietäten in Deutschland und/oder Österreich interessant wäre.
} 
(mit über $70 \%$ der Belege) bevorzugt, während die Dialekte die syntaktisch und/oder konzeptuell "aufwändigeren" Varianten (in über $80 \%$ der Fälle) bevorzugen. Ich habe an anderer Stelle (Berthele 2004) ausführlicher nachgewiesen, dass komplexere Fügungen sowohl in statischen als auch in dynamischen Raumausdrücken ganz generell typisch sind für die alemannischen Dialekte, und dass diese Unterschiede auch statistisch signifikant sind. Als sehr verkürzter Beleg für diese Aussage soll Abb. 1 dienen, die die Mittelwerte des Gebrauchs von erweiterten $(\mathrm{PP}+\mathrm{ADV})$ vs. einfachen (PP alleine) Konstruktionen in den untersuchten Dialekten wiedergibt.

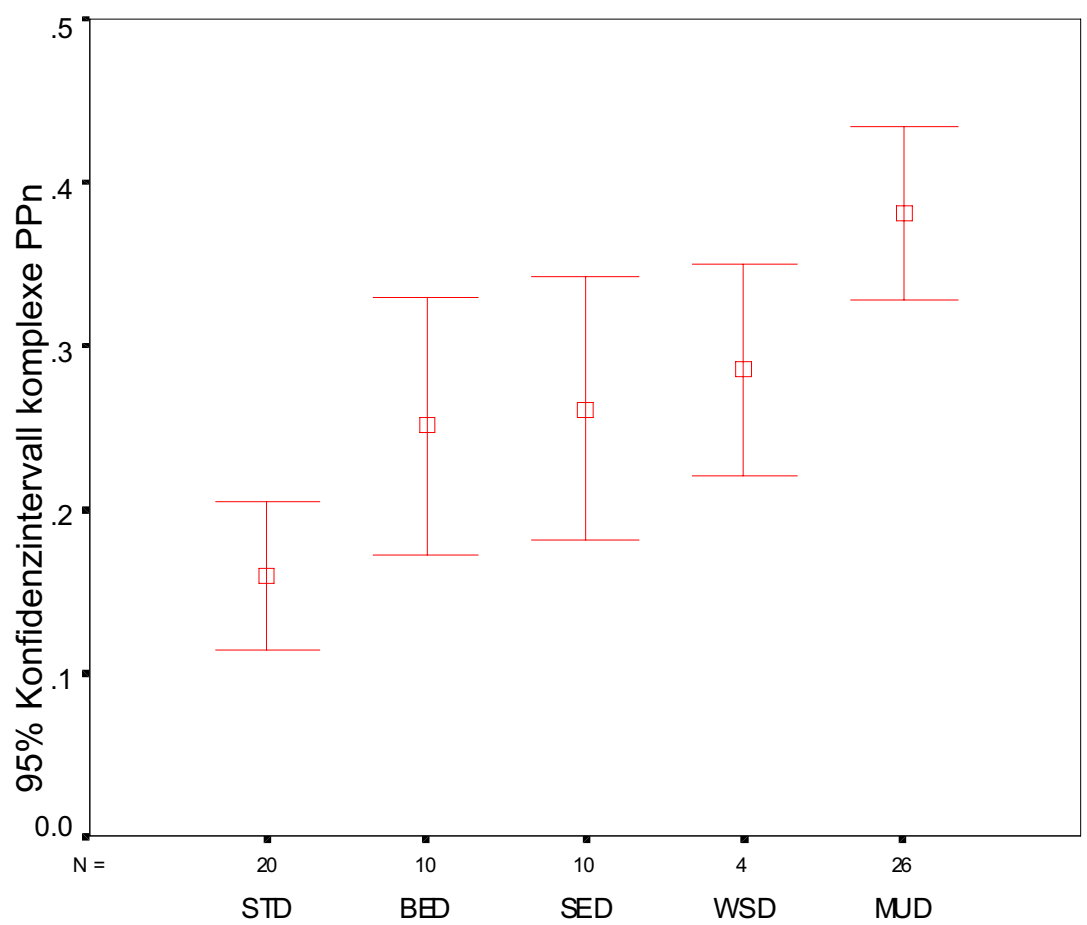

Abb. 1: Mittelwerte und Konfidenzintervalle für die Anteile komplexer Wegangaben pro Varietät ${ }^{13}$

Abb. 1 weist zudem auf einen weiteren Zusammenhang hin, der statistisch hochsignifikant ist: Nich alle dieser Dialekte sind gleich gross, was ihre (geschätzte) SprecherInnenzahl angeht. Und je kleiner ein Dialekt ist, desto stärker tendiert er zum offenen Ausdruck der Raumrelationen. ${ }^{14}$ Eine analoge Untersuchung dreier rätoromanischer Idiome, lässt den Schluss zu, dass auch hier eine Tendenz zu verteilter Raumsemantik besteht (Berthele [im Druck, in Vorbereitung]). Das hier herausgegriffene Beispiel des "Fenstersturzes" illustriert also einen generellen Trend, einen Trend, der auch der traditionellen einigen Dialektologie nicht entgangen ist (Zinsli 1945: 177 und 257, Szadrowsky 1930: 79; Idiotikon 2,1319).

\footnotetext{
13 Die Zürichdeutschdaten fehlen in dieser Abb., sie sind nicht Teil des in Berthele (in Vorbereitung) ausgewerteten Materials, wurden speziell für den vorliegenden Aufsatz hinzugezogen und konnten lediglich auf die Fensterszene hin ausgewertet werden.

14 Die Grösse der Dialekte wurde aufgrund von Volkszählungsdaten geschätzt, es wurde eine Rangskala von 1 bis 4 errechnet ( $\mathrm{n}<10^{\prime} 000$ : 1; 10'000< $\mathrm{n}<100^{\prime} 000$ : 2; 100'000< $\mathrm{n}<1^{\prime} 000^{\prime} 000$ : 3; $\mathrm{n}>1$ 000'000: 4). Die so entstandene Skala ergibt eine hochsignifikante Korrelation mit der abhängigen Variable (spearman's rho Koeffizient).
} 
Es wird im Folgenden abschliessend zu diskutieren sein, inwiefern die von den Dialekten bevorzugten Konstruktionen tatsächlich konzeptuell komplexer sind, und wie die hier vorgefundenen Variationsmuster erklärt oder zumindest verstanden werden könnten.

\section{Wohin ist der Hund geflogen - bzw. wo ist das Wegkonzept genau untergebracht?}

Es ist mittlerweile unbestritten (cf. Sinha/Kuteva 1995: 169; Bennett 1993), dass es zum Erfassen der relationalen räumlichen Bedeutung eines Ausdrucks nicht reicht, einzelne, vorzugsweise geschlossene Formklassen wie etwa $\mathrm{P}$ durch kontrastive Analysen zu beschreiben. $\mathrm{Ob}$ beispielsweise die deutsche $\mathrm{P}$ in einen Ort oder eine Richtung angibt, hängt einerseits vom von in regierten Kasus ab, aber auch oft vom (Bewegungs)Verb:

\section{3 die Banditen flohen in den Wald \\ 24 ?die Banditen flohen im Wald \\ 25 die Banditen brieten Würste im Wald}

Es ist daher oft angemessen, die direktionale oder lokale Bedeutung eines Ausdrucks nicht einfach einer $\mathrm{P}$ zuzuschreiben, sondern dem ganzen Ausdruck (etwa im Sinne einer Konstruktion der Construction Grammar (Goldberg 1995, Fillmore/Kay 1999). Aspekte der räumlich-relationalen Semantik sind auf verschiedene syntaktische Elemente aus verschiedenen Formklassen (offenen und geschlossenen) verteilt. Dies können wir mit Sinha/Kuteva (1995) als verteilte Raumsemantik bezeichnen. Die AutorInnen postulieren, dass alle Sprachen dieses Phänomen kennen (173). Bei der sprachvergleichenden Untersuchung dieser verteilten Raumsemantik ist es sodann das Ziel, die unterschiedliche Gewichtung der einzelnen beteiligten Formklassen herauszuarbeiten. Sinha und Kuteva zeigen beispielsweise, wie im Niederländischen die Verben für den Ausdruck der Raumrelationen wichtiger sind als etwa im Englischen (zu beobachten etwa im Bereich der Positionsverben zitten, staan, etc.). Auf die im vorliegenden Beitrag untersuchten Konstruktionen übertragen lässt sich also feststellen, dass die Varietäten des Schweizerdeutschen innerhalb der VP beträchtliches Gewicht auf die Richtungsadverbien legen. Diese Adverbien können einerseits einfach den Weg der P wiederholen, sehr oft fügen sie jedoch einen zusätzlichen Aspekt hinzu (in unseren Konstruktionen vor allem den vertikalen Weg und den raus-Weg).

In den Varietäten des Schweizerdeutschen wird der begriffliche Kern des Bewegungsereignisses, also der Weg, den die Figur durch das Fenster zurücklegt, auf verschiedene syntaktische Elemente verteilt. In der Regel handelt es sich um zwei "Mikro"Wege der "Makro"-Bewegung. Der erste Teil des Weges ist dabei oft auf die Quelle, den Ausgangsort der Bewegung bezogen (ausgedrückt in $\mathrm{P}$ wie von oder $a b$ ), oder er kann einen durch einen bestimmten Meilenstein gekennzeichneten Weg ausdrücken ( $\mathrm{P}$ durch). Es zeigt sich dabei auch hier wieder (cf. Langacker 1999: 55f.; Smith 1987), dass im Deutschen der Dativ oft mit dem Quelle-Weg Abschnitt des Quelle-Weg-Ziel-Schemas identifiziert werden 
kann (aus dem Fenster, vom Fensterbrett), während der Akkusativ in der Regel mit dem Weg-Ziel Abschnitt zusammenhängt (aus dem Fenster auf den Boden).

In zwei Typen wird der Weg nicht in zwei Abschnitte zerlegt, sondern das MakroBewegungsereignis wird zuerst an einem Ort situiert (mittels $z u$ oder bei), und in einem zweiten Schritt wird die Figur auf einem aus-Weg konzeptualisiert.

In der Analyse oben wurden zwar die Bewegungsverben nicht explizit behandelt, es liegt aber auf der Hand, dass auch diese einen Beitrag zur verteilten Raumsemantik leisten: Verben wie fallen (schwdt. khiien, gheien, etc., vgl. Bsp. 26) implizieren, dass der Weg in der Vertikalen und abwärtsgerichtet verläuft. Ich schlage deshalb vor, Konstruktionen wie in Bsp. 26 wie in 27 gezeigt zu verstehen. da auf ein Mal ist da der Hund vom Fenstersims hinunter gefallen

\begin{tabular}{|c|c|c|}
\hline $\begin{array}{l}\mathrm{r} H \mathrm{u} \\
\text { isur }\end{array}$ & & om Fenstersims \\
\hline
\end{tabular}

Die verschiedenen syntaktischen Elemente, die einen Beitrag zur Assoziationsfunktion leisten, entsprechen in der in Bsp. 26 wiedergegebenen Konstruktion unterschiedlichen Konzepten. Während das Verb für einen abwärtsgerichteten Weg steht, gibt die Präposition einen dimensional nicht spezifizierten Weg weg von der in der NP angegebenen Quelle an, und das Adverb schliesslich wiederholt den Abwärtsweg.

Es muss hier nochmals gesagt werden, dass die Unterschiede zwischen den Mundarten und der Standardsprache grundsätzlich nicht einem Gegensatz von grammatisch/ungrammatisch entsprechen (viele der dialektalen Konstruktionen würden wohl von Standardsprechern nicht als falsch, sondern einfach als ungewöhnlich oder nicht zu ihrem Repertoire gehörend kategorisiert), sondern Unterschiede in der Frequenz sind. Die Daten- und Korpusanalyse hat klar gezeigt, dass die lokalisierenden VP im Bereich der hier betrachteten Szene im Schweizerdeutschen in der Regel ein Adverb beinhalten. Die Klasse der Richtungsadverbien hat also in diesen Varietäten ein viel grösseres Gewicht als in der Standardsprache. Dabei dient sie sowohl dem differenzierenden Ausdruck von Teilaspekten des zusammengesetzten Weges als auch dem nochmaligen Ausdruck des schon im V und/oder in der $\mathrm{P}$ Ausgedrückten.

Dieser Frequenzunterschied zeigt nicht nur, dass ein Unterschied in Gewichtung und Einbezug der verschiedenen Wortarten besteht, sondern auch, dass das Schweizerdeutsche die Raumsemantik offener, sichtbarer ausdrückt als die Standardsprache. Dies entspricht genau der von Sinha und Kuteva beim Vergleich von anderen Sprachen gefundenen Variation:

Languages vary in terms of the extent to which the underlying distributed semantics of space is manifest or overt in expression. (Sinha/Kuteva 1995: 1973)

In so unterschiedlichen Sprachen wie Japanisch und Bulgarisch finden sich offenbar Konstruktionen, die auf ganz ähnliche Weise wie in den hier beschriebenen 
schweizerdeutschen Formen in verschiedenen Teilen des Syntagmas komplementäre und redundante Aspekte des Wegs/Orts ausdrücken (Sinha/Kuteva 1995: 186ff.).

Im vorliegenden Beitrag wurde exemplarisch gezeigt, dass die Varietäten des Schweizerdeutschen insgesamt zu einem offeneren Ausdruck der Raumrelationen neigen. Ausserdem wurden unterschiedliche Varianten des Weg-Ausdrucks verglichen, und es zeigte sich, dass dieselben Bilder auf dem Papier offenbar recht unterschiedliche Konzeptualisierungen in den Köpfen auslösen. Inwiefern einzelne dieser FenstersturzKonstruktionen Idiosynkrasien sind, müsste eine grössere Datenerhebung an den Tag bringen. Da jedoch ausser in einem Zweifelsfall analoge Konstruktionen auch bei Perzeptionsverben und Verben des Sprechens gefunden wurden, können wir davon ausgehen, dass hier nicht einfach nur ad-hoc-Bildungen vorliegen. Die Beispiele sollten also gezeigt haben, dass sich der Hund zwar für alle Gwpp. von einem Ort auf der Innenseite des Fensters an einen Ort auf der Aussenseite des Fensters bewegt, die konzeptuelle Erfassung des dabei durchlaufenen Weges jedoch beträchtlich variieren kann. Es zeigt sich - gleichsam als ceterum censeo der Variationslinguisten - auch in diesem Bereich, dass die sprachvergleichende Untersuchung in der kognitiven Semantik gut daran tut, nicht nur Standardvarietäten zu betrachten, sondern auch die dialektale Variation in ihre Untersuchungen einzubeziehen.

\section{Literaturangaben}

Becker, Angelika (1994): Lokalisierungsausdrücke im Sprachvergleich: eine lexikalischsemantische Analyse von Lokalisierungsausdrücken im Deutschen, Englischen, Französischen und Türkischen. Tübingen.

Berthele, Raphael (im Druck): "Spatial Reference in an Endangered Romance Language: The Case of Romansh". Proceedings of the Berkeley Linguistics Society (BLS) 2003.

Berthele, Raphael (in Vorbereitung): "Ort und Weg. Eine vergleichende Untersuchung der sprachlichen Raumreferenz in Varietäten des Deutschen, Rätoromanischen und Französischen".

Berthele, Raphael (2004): "The typology of motion and posture verbs: A variationist account". In: Kortmann, Bernd (ed.): Dialectology Meets Typology. Dialect Grammar from a Cross-Linguistic Perspective. Berlin/New York: 93-126.

Duden (1994): Das grosse Wörterbuch der deutschen Sprache in acht Bänden. Mannheim/ Leipzig/Wien.

Eisenberg, Peter (et al.) (1998): Duden Grammatik. 6. Auflage. Mannheim/Leipzig/Wien/ Zürich.

Fillmore, Charles J./Kay, Paul (1999): "Grammatical constructions and linguistic generalizations: The 'What's X doing Y?' construction". Language 75/1: 1-33.

Goldberg, Adele (1995): Constructions. A Construction Grammar Approach to Argument Structure. Chicago.

Hinderling, Robert (1980): "Die Richtungsadverbien im Bayerischen und im Alemannischen. Versuch einer Synopse". In: Rowley, A. (1980): 249-296. 
Idiotikon (1881ff.): Schweizerisches Idiotikon. Wörterbuch der schweizerdeutschen Sprache. Frauenfeld.

Johnson, Mark (1987): The Body in the Mind: The Bodily Basis of Meaning, Imagination, and Reason. Chicago/London.

Lakoff, George/Johnson, Mark (1999): Philosophy in the flesh. New York.

Langacker, Ronald W. (1990): Concept, Image, and Symbol. The Cognitive Basis of Grammar. Berlin/New York.

Langacker, Ronald W. (1999): Grammar and Conceptualization. Berlin/New York.

Levinson, Stephen C. (1996): "Language and Space". Annu.Rev. Anthropol. 25: 353-382.

Mayer, Mercer (1969): Frog, Where Are You? New York.

Niemeier, Susanne/Dirven, René (eds.) (2000): Evidence for Linguistic Relativity. Amsterdam/Philadelphia.

Olsen, Susan (1996): "Pleonastische Direktionale". In: Harras, Gisela/Bierwisch, Manfred (eds.): Wenn die Semantik arbeitet: Klaus Baumgärtner zum 65. Geburtstag. Tübingen: 303-328.

Özcaliskan, Seyda/Slobin, Dan I. (1999): "Learning How to Search For the Frog: Expression of Manner of Motion in English, Spanish, and Turkish". In: Greenhill, Annabel/Littlefield, Heather/Tano, Cheryl (eds.): BUCLD 23: Proceedings of the 23rd annual Boston University Conference on Language Development. Volume II. Boston: 541-552.

Rowley, Anthony (ed.) (1980): Sprachliche Orientierung1: Untersuchungen zur Morphologie und Semantik der Richtungsadverbien in oberdeutschen Mundarten. Bayreuth.

SDS: Baumgartner, Heinrich et al. (1962-1997): Sprachatlas der deutschen Schweiz. Bern/ Basel.

Sinha, Chris/Kuteva, T. (1995): "Distributed spatial semantics". Nordic Journal of Linguistics 18: 167-199.

Slobin, Dan I. (1991): "Learning to think for speaking. Native language, cognition, and rhetorical style". Pragmatics 1: 7-26.

Slobin, Dan I. (1996): "Two ways to travel: Verbs of motion in English and Spanish". In: Thompson, Sandra A./Shibatani, Masayoshi (eds.): Grammatical constructions: Their form and meaning. Oxford: 195-217.

Smith, Michael B. (1987): The semantics of dative and accusative in German: An investigation in cognitive grammar. San Diego.

Steinitz, Renate (1969): Adverbial-Syntax. Berlin.

Szadrowsky, Manfred (1930/1936/1937): "Zur hochalemannischen Syntax", I-V. Beiträge zur Geschichte der deutschen Sprache und Literatur 54, 60, 61. PBB 54 (1930): 65-137 [I-II]; 54 (1930): 281-293; 60 (1936): 445-458; 61 (1937): 273-288.

Talmy, Leonard (1985): "Lexicalization patterns: semantic structure in lexical forms". In: Shopen, Timothy (ed.): Language typology and syntactic description. Volume III: Grammatical categories and the lexicon. Cambridge/London etc.: 57-149.

Talmy, Leonard (2000a): Toward a cognitive semantics. Volume I: Concept Structuring Systems. Cambridge, Massachusetts/London, England.

Talmy, Leonard (2000b): Toward a cognitive semantics. Volume II: Typology and process in concept structuring. Cambridge, Massachusetts/London, England: MIT Press. 
Werlen, Iwar (2002): Sprachliche Relativität. Basel/Tübingen.

Wunderlich, Dieter (1984): "Zur Syntax der Präpositionalphrasen im Deutschen". Zeitschrift für Sprachwissenschaft 3/1: 65-99.

Zinsli, Paul (1945): Grund und Grat: die Bergwelt im Spiegel der schweizerdeutschen Alpenmundarten. Bern. 